\title{
Communication structure and the locus of the reinforcing function of speaking in reply
}

\author{
ROBERT FRANK WEISS, JOYCE JETTINGHOFF WEISS, \\ MICHELE K. STEIGLEDER, and ROBERT E. CRAMER \\ University of Oklahoma, Norman, Oklahoma 73019
}

\begin{abstract}
The communication structure of experiments on the reinforcing function of speaking in reply is designed to permit the subject to reply to the other person while rigorously excluding information feedback, and consequently it is unlikely that a social influence mechanism could be the locus of the reinforcing effect. It is possible to design a communication structure which increases the opportunity for social influence within the no-feedback structural constraint. An experimental group having such a structure $(\mathrm{N}=50)$ was compared with a control group which followed the standard reply procedure $(\mathrm{N}=50)$, and the results unambiguously confirmed that the locus of the reinforcement effect does not lie in social influence or the opportunity for social influence.
\end{abstract}

A structural difference between the monologue of mass communications and the dialogue of personal communication is the opportunity of each participant to reply to the other. Replying plays a fundamental functional role in interpersonal communication rather than serving only as a mere dependent variable or as an influencer of one participant by the other. People will learn an instrumental response the reinforcement for which is the opportunity to speak in reply. A continuing series of experiments reveals a striking pointfor-point correspondence between the effects of reinforcement in discrete-trials instrumental escape conditioning and the effects of speaking in reply in conversation. Experimental demonstration of our theoretical analogies extends well beyond the analogs of acquisition and extinction, commonly deemed sufficient in social psychology or behavior therapy, to include analogs of partial reinforcement effects, intermittent shock effects, delay of reinforcement effects, drive effects, correlated reinforcement effects (discretetrials DRL), and correlated delay of reinforcement effects (e.g., Weiss, Boyer, Colwick, \& Moran, 1971; Weiss, Lombardo, Warren, \& Kelley, 1971). These experiments indicate that a basic form of interpersonal communication resembles escape rather than reward conditioning: The motivation is aversive rather than appetitive and speaking in reply functions as a negative reinforcement, like shock termination, rather than as a positive reinforcement, like food. Moreover, two experiments investigating analogs of intermittent shock appear to pinpoint the locus of the drive in the other person's initial disagreeing opinion. We are interested in further determining the locus of drive and reinforcement effects and have conducted several experiments in

Reprints may be obtained from R. F. Weiss, Department of Psychology, University of Oklahoma, Norman, Oklahoma 73019. order to do so. In escape conditioning the effects of drive are manifested in the ability of drive to motivate instrumental escape learning and reduction of the noxious drive to instrumentally reinforce escape learning. Learning theory ascribes other properties to noxious drives besides the ability of drive reduction following an instrumental response to reinforce. These other properties of drive can be manifested in learning paradigms other than escape conditioning. Two such paradigms are energization by irrelevant drive (for which paired associate methods have been widely employed) and classical conditioning of a learned drive, and our research employing both these experimental paradigms further points to the other person's initial disagreement as the locus of the aversive drive (Lombardo, Libkuman, \& Weiss, 1972; Weiss, Note 1). Similarly, learning theory ascribes other properties to drive reduction besides the ability of drive reduction following an instrumental response to reinforce. These properties of drive reduction can be manifested in paradigms other than instrumental escape conditioning. While the experiments modeled on escape conditioning provide extensive evidence for replying as a locus of reinforcement, we have been able to provide particularly clear evidence for the reduction of aversive drive through replying via the attraction paradigm and reduction of irrelevent drive energization of paired associate learning (Lombardo, Weiss, \& Stich, 1973; Weiss, Note 1).

By drawing on learning paradigms other than escape conditioning to supplement our extensive research with the escape paradigm, we have been able to make effective use of the very considerable resources of learning theory. A particular virtue of our research has been its theoretical elegance. Substantively, however, this research takes its rise in the investigation of the functional significance of a fundamental structural aspect of communication, and it is in keeping with this enterprise that we now, raise further questions concerning the 
locus of the reinforcing function of speaking in reply. In our studies of the reinforcing function of speaking in reply, the communication structure of the experiment was designed to permit the subject to reply to the other person while rigorously excluding information feedback from the other person or anyone else (e.g., consensual validation, social support). When information feedback is carefully and deliberately withheld, to whom is the reply addressed? Novel experimental manipulation of the communication structure of the reply experiment can serve to illuminate fundamental aspects of the communication structure of a typical reply experiment and the locus of the reinforcing function of the reply. In our first such experimental manipulation of the communication structure (Weiss, Note 1), we were able to show that the other person is an essential component of the communication structure and that the subjects were not merely venting their spleens, justifying themselves to themselves, or musing philosophically upon the issues at hand. Modification of the communication structure made completely explicit what could be inferred from the results of various experiments which manipulated reinforcement contingencies such as extinction. It is also reasonable to infer from demonstrations of the reinforcing effects of speaking in reply in the absence of information feedback from others that the locus of the reinforcing effect does not lie in a social influence mechanism. It would, however, be worthwhile to explicitly determine whether a social influence or proselytization mechanism could be operative in our experimental situation even in the absence of such information feedback.

\section{METHOD}

\section{Logic and Design}

On each trial of a reply experiment, the subject listens to another person's viewpoint and can then press a switch (the instrumental response), the reinforcement for which is speaking in reply to the other person. In this experiment such a typical reply experimental communication structure was compared with a three-person communication structure in which the subject had the opportunity to address someone in addition to the person who addressed him. If social influence, an opportunity for social influence, or proselytization underlies the reinforcing effect of speaking in reply, then two persons afford more opportunity for social influence than one and it should be more reinforcing to proselytize two people instead of only one. We have already noted that inference from the results of our previous experiments indicates that the reply experiments are not concerned with social influence flows and that, therefore, the increased opportunity for social influence inherent in the threeperson communication structure should not affect the reinforcing function of speaking in reply. Particular assumptions about the underlying nature of drive and reinforcement (e.g., Byrne, 1971; Dollard \& Miller, 1950; Weiss, Lombardo, Warren, \& Kelley, 1971) also permit a prediction of no difference between these two experimental conditions. It seems worth emphasizing that the formal escape conditioning model, in its purity, does not address this kind of question.

\section{Deception and Masking Task}

The experiment was represented to the subjects as a study of opinion change. "We are interested in how your opinion may be affected by what someone else says, how your opinion may be affected by what you yourself say, and how what you say may affect the opinions of someone else." As a masking task, after each statement and reply, the subject indicated whether he had changed his opinion by moving a dial. Congruent with this deceptive rationale of attitude change through conversation, which is standard procedure in our previous reply experiments, the additional person in the three-person communication structure "listened" to both the other person's taped initial statements and the actual subject's reply without himself speaking. Both the other person who spoke with the subject and the additional person were said to be in other rooms from the subject, as is standard procedure in the reply experiments. Since the additional person in the three-person communication structure did not speak in the conversation, elaborate measures were taken to impress his presence vividly upon the subjects in that condition. These measures included instructions to the additional person, instructions to the subject and the other person not to wait for the additional person to speak, since he was supposed merely to silently register his opinion change, and a tape-recorded question from the additional person about the instructions. As a continuing reminder on each trial throughout the experiment, a pair of labeled lights were added to the subject's part of the apparatus, one for the other person and one for the additional person, which lit up to indicate that the subject's intercom was connected to both of the two persons whenever he spoke in reply. The opportunity for proselytization of the additional person was made salient by opinion-change rationale: "In the case of Subject Orange [the additional person], we are interested solely in the effect on his opinions of what he hears. People often do form opinions simply by listening to what others have to say."

\section{Apparatus and Procedure}

The subject was seated at a table facing the control room wall, which included four one-way vision windows. Instructional signals appeared in each window upon illumination. The signals were the large printed words (1) "listen," (2) "throw switch if you wish to comment," (3) "talk," and (4) "move dial to final opinion." A panel mounted on the table top contained the subject's "comment" switch, his intercom, and the maskingtask opinion-change dial.

An experimental trial began with the "listen" signal and the playing of the taped topic and opinion by the other person. When the taped message ended, the experimenter operated the control which simultaneously (1) presented the CS, the signal "throw switch if you wish to comment," and (2) started the latency timer. When the subject threw the comment switch, the latency timer automatically stopped, measuring latency to $.01 \mathrm{sec}$. The "talk" signal followed the switch-press response and the subject spoke in reply. The procedure described was closely modeled on traditional discrete-trials instrumental conditioning. The reinforcement (speaking in reply) was contingent on the instrumental response (switch pressing). The dependent variable was speed (100/latency), measured from the time of the presentation of the CS (signal "press switch if you wish to comment") to the instrumental switch-pressing response.

\section{Subjects and Materials}

The subjects were 100 undergraduates from the introductory psychology pool, 50 in each condition. The experiment was counterbalanced for sex, even though previous research indicated no main or interactive effects of sex (with the present experiment confirming this).

The opinions expressed by the "other person" were selected by means of a questionnaire administered to 100 subjects from the introductory psychology pool. Selection criteria were that the opinions should be reasonably consistent with each other and dissimilar from the opinions of the introductory psychology students. The "other person" cassette tapes were the same sex as the subject, had a moderate regional accent characteristic of in-state students, were carefully gauged to approximate the 
articulateness and sophistication of a typical subject, and were presented in randomized order.

\section{RESULTS AND DISCUSSION}

In both experimental communication structures, the subjects learned the instrumental response. Instrumental response speed increased gradually over the 12 acquisition trials, with the two groups showing almost identical speeds throughout the course of learning. The acquisition effect was highly significant $[F(11,1056)=4.11$, $\mathrm{p}<.001]$, while differences between the two communication structures were almost "significantly nonsignificant" in their main effect $(F<1)$ and interaction with acquisition trials $(\mathrm{F}<1)$. The locus of the reinforcing effect does not lie in social influence or in the opportunity for social influence. Replying plays a fundamental functional role in interpersonal communication commensurate with its structural significance.

\section{REFERENCE NOTE}

1. Weiss, R. F. Interpersonal communication: The reinforcing function of speaking in reply. Draft preprint,
University of Oklahoma, 1976. Available from R. F. Weiss, Department of Psychology, University of Oklahoma, Norman, Oklahoma 73019.

\section{REFERENCES}

BYRNE, D. The attraction paradigm. New York: Academic Press, 1971.

Dollard, J., \& Miller, N. E. Personality and psychotherapy. New York: McGraw-Hill, 1950.

Lombardo, J. P., Libkuman, T. M., \& Weiss, R. F. The energizing effects of disagreement-induced drive. Journal of Experimental Research in Personality, 1972, 6, 133-141.

Lombardo, J. P., Weiss, R. F., \& Stich, M. H. Effectance reduction through speaking in reply and its relation to attraction. Journal of Personality and Social Psychology, 1973, 28, 325-332.

Weiss, R. F., Boyer, J. L., Colwick, J. T., \& Moran, D. J. A delay of reinforcement gradient and correlated reinforcement in the instrumental conditioning of conversational behavior. Journal of Experimental Psychology, 1971, 90, 33-38.

Weiss, R. F., Lombardo, J. P., Warren, D. R., \& Kelley, K. A. The reinforcing effects of speaking in reply. Journal of Personality and Social Psychology, 1971, 20, 186-199.

(Received for publication November 13, 1976.) 